\title{
Rationale and Study Protocol for a Two-Part Intervention: Safety Planning and Structured Follow-Up among Veterans at Risk for Suicide and Discharged from the Emergency Department
}

Article CONCLI_1189

Title: Rationale and Study Protocol for a Two-Part Intervention: Safety Planning and Structured Follow-Up among Veterans at Risk for Suicide and Discharged from the Emergency Department

Authors with Academic Affiliations, in order from first to last

Glenn W. Currier, M.D., M.P.H.

Department of Psychiatry, University of Rochester School of Medicine, Rochester, NY

Department of Psychiatry \& Behavioral Neurosciences, University of South Florida, Tampa, FL

Gregory K. Brown, PhD

Department of Psychiatry

Perelman School of Medicine of the University of Pennsylvania

Philadelphia, PA

Lisa A. Brenner, Ph.D.

Rocky Mountain Mental Illness Research Education and Clinical Center, Denver, CO

Departments of Physical Medicine and Rehabilitation, \& Neurology

University of Colorado, Anschutz Medical Campus, Aurora CO

Megan Chesin, $\mathrm{PhD}$

Department of Psychiatry, Columbia University and New York State Psychiatric Institute

New York, NY

Kerry L. Knox, PhD

Department of Psychiatry, University of Rochester School of Medicine, Rochester, NY

Department of Veterans Affairs, VISN 2 Center of Excellence for Suicide Prevention, Canandaigua, NY

Marjan Ghahramanlou Holloway, Ph.D. 
Departments of Medical \& Clinical Psychology and Psychiatry

Uniformed Services University of the Health Sciences

Bethesda, MD

Barbara Stanley, PhD

Department of Psychiatry, Columbia University and New York State Psychiatric Institute

New York, NY

\begin{abstract}
There are no evidence-based, brief interventions to reduce suicide risk in Veterans. Death by suicide is a major public health problem. This article describes a protocol, Suicide Assessment and Follow-up Engagement: Veteran Emergency Treatment [SAFE VET], developed for testing the effectiveness of a brief intervention combining a Safety Planning Intervention with structured follow-up (SPI-SFU) to reduce near-term suicide risk and increase outpatient behavioral health treatment engagement among Veterans seeking treatment at Veteran Affairs Medical Center (VAMC) emergency departments (EDs) who are at risk for suicide. In addition to describing study procedures, outcome measures, primary and secondary hypotheses, and human subjects' protection issues, the rationale for the selection of SPI-SFU as the intervention is detailed, as are safety considerations for the unique study setting and sample.
\end{abstract}




\section{Introduction}

Suicide is the $10^{\text {th }}$ leading cause of death in the United States. In 2012, suicide claimed over 40,000 lives [1]. For two decades (from the mid- to late 1980 s to the mid- to late 2000s), suicide rates among Veterans were higher than rates among their same aged and sex civilian peers [2-4]. In subsequent years, with the implementation of additional Department of Veterans Affairs (VA) initiatives to prevent suicide among Veterans, decreasing proportions of Veterans among U.S. suicide victims have been observed [5]. Nonetheless, suicide is still a major public health problem among U.S. Veterans. In 2010, suicide claimed an estimated 22 Veteran lives each day [5]. Non-fatal suicide attempts and serious suicidal ideation occur much more frequently [6] and confer particular risk for subsequent suicide in both U.S. civilian and Veteran populations [7].

Emergency departments (EDs), both within Veterans Health Administration (VHA) and other healthcare systems nationally, are assuming an increasingly important role in the care of suicidal individuals. In fact, suicide-related ED visits increased nearly 50\% from 1992-2001 [8]. In 2010 and 2011, there were an estimated 2.5 million suiciderelated ED-visits annually in the U.S. [9]. Given limits to outpatient treatment engagement among populations at risk for suicide [10, 11], EDs often function as the primary or sole point of contact within the health care system for such individuals [12]. This contact often occurs either immediately following a suicide attempt or when suicidal thoughts escalate, and the individual feels in danger of acting on them.

Although American Psychiatric Association Clinical Practice Guidelines have been established for conducting suicide risk assessments [13], there are no widely adopted best practices for treating suicidal patients within emergency settings [14]. In 
practice, when suicidal patients are evaluated in the ED and hospitalization is not clinically indicated, they are generally discharged with referrals for outpatient mental health treatment [15]. Unfortunately, between $40 \%$ and $90 \%$ of suicidal patients assessed and then discharged from the ED do not attend even 1 follow-up outpatient mental health care appointment [16-19]. Of those who do attend some treatment, lags between ED discharge and first outpatient visit, as well as premature termination of outpatient treatment, are common and concerning. Only between $10 \%$ and $40 \%$ of suicidal ED patients discharged with a referral for outpatient mental health treatment are compliant with recommended treatment over 3 to 12 month follow-up [17-19]. In the 6-month period following discharge from an ED visit for self-harm (suicide intent unknown) suicide rates are 562 per 100,000 individuals [20], whereas the suicide rate in the general U.S. population is 12.6 per 100,000 [21]. Because many patients will not seek or remain in treatment following an emergency evaluation or receive services in a timely manner, and given the increased suicide risk associated with the period following ED presentation for self-directed violence, provision of a brief, targeted psychosocial intervention to prevent suicide delivered in the ED is critical.

Thus, a multi-faceted approach combining the Safety Planning Intervention [19] and structured follow-up (SPI-SFU) was selected for implementation as a suicide prevention intervention for all patients presenting for clinical care in five different VAMC EDs in a project named Suicide Assessment and Follow-up Engagement: Veteran Emergency Treatment [SAFE VET]. The associated clinical demonstration project is described elsewhere [22]. Interpretation of outcomes from the clinical demonstration project was limited by a lack of a control condition. Patient reported outcomes were also 
not extensively tracked after ED discharge. However, the introduction of the SAFE VET intervention in some, but not all, VAMC EDs provided an opportunity for a quasiexperimental cohort comparison design. Since patient-level randomization was not feasible within the intervention sites where all eligible patients were receiving the SPI, we took advantage of the staggered roll-out of ED involvement to compare four of the intervention sites with four other VAMC EDs with similar patient flow characteristics and where the SAFE VET intervention was not provided. An adapted version of this intervention is also being tested in a concurrent randomized controlled trial that is ongoing in a military cohort who is being treated in an inpatient psychiatric setting [23].

The SPI is a brief intervention in which the patient and provider collaboratively develop a written, individualized list of coping skills to use, and professional and social supports to contact in suicidal crisis. SPI has been successfully implemented in a variety of settings including acute care, inpatient units, and suicide hotline service centers. The SFU portion of the intervention is designed specifically for SAFE VET and provides post-ED-discharge telephone contact between the patient and a clinician working for the project, usually the one who completed the safety plan with the patient.

\subsection{Study aims and hypotheses}

The primary aim of this study is to determine the effectiveness of SPI-SFU compared to usual ED clinical care, augmented by additional assessment (E-CARE), for decreasing suicide behaviors, suicide ideation and increasing treatment engagement and suicide-related coping strategies in Veterans seen in the ED for a suicide-related concern (i.e., significant suicide ideation or behavior) who do not require an inpatient hospitalization. We hypothesize that Veterans receiving SPI-SFU versus E-CARE will: 
1) be less likely to attempt suicide within 6 months of the index ED visit;2) have less severe suicidal ideation at 6 months post-index ED visit; 3) have greater suicide-related coping abilities at 6-months post-index ED visit, and 4) be more likely to attend 1 or more outpatient mental health or substance abuse treatment appointments within 6 months following the index ED visit.

\section{Methods}

\subsection{Design}

The study uses a quasi-experimental design to assess the effectiveness of SPISFU to E-CARE by comparing outcomes from 4 VAMC EDs where SPI-SFU is routinely carried out to outcomes from 4 similar EDs where the intervention is never employed. Eligible patients who present for ED care during day and evening shifts are invited to participate. Research assessments are administered at baseline and at 1,3, and 6 months after the index ED visit (See Figure 1). The trial is registered with ClinicalTrials.gov (identifier: NCT01334541).

\subsection{Setting}

Four VAMC EDs where SPI-SFU is already implemented as part of standard care for suicidal patients serve as the intervention sites: Portland, Denver, Manhattan, and Philadelphia VAMCs. Four matched control sites where SPI-SFU components are not provided are also participating. Matching criteria include urban/suburban versus rural setting, similar number of psychiatric ED evaluations provided per year, and presence of a psychiatric inpatient unit within the same VAMC. The control sites are Long Beach, Bronx, Milwaukee, and San Diego VAMCs. The eight participating VAMC EDs each 
treat an average of approximately 10,000 patients per year, of which about $10 \%$ present for mental health or substance abuse treatment purposes.

\subsection{Recruitment and informed consent}

Eligible Veterans, who appear to meet study criteria (see Section 2.4.1) and are deemed to be appropriate for the study by treating ED physician, are referred to research staff for enrollment. Patients are considered appropriate for inclusion if the treating clinician does not consider it clinically contra-indicated. Study staff then meet with patients in the ED to determine eligibility, explain the study purpose, risks and benefits, and procedures, answer any questions about the study, and sign the VA Institutional Review Board (IRB)-approved study consent form and the Health Insurance Portability and Accountability Act (HIPAA) authorization form. All research team members hold a Bachelor's degree or higher in Psychology or a related field. The information for consented patients is tracked based on the guidance provided by the Consolidated Standards of Reporting Trials (CONSORT) statement [www.consort-statement.org].

\subsection{Participants}

Based on a power analysis (see section 2.12.4) a minimum of 300 Veterans per treatment condition are expected to be recruited for this study. Study inclusion and exclusion criteria are provided below.

\subsubsection{Inclusion and exclusion criteria}

Inclusion criteria are as follows: 1) Veteran receives the Safety Planning Intervention at VA SAFE VET EDs or receives treatment-as-usual at VA control site EDs; 2) aged 18 years or older; 3) identified as being at risk for suicide based upon presenting complaints and/or the assessment of an ED clinician; 4) able to provide 2 
collateral contacts with telephone numbers for tracking purposes; 5) in a stable living situation (i.e., able to provide a residential, domiciliary or shelter address at study entry); and, 6) able to provide a home, cellular, or other telephone number where the participant can be reached.

Exclusion criteria are as follows: 1) unable to read and understand English; 2) unable or unwilling to give informed consent as determined either by ED clinical staff or study staff; and/or 3) admitted to the VAMC inpatient psychiatric unit from the ED. All patients who meet study entry criteria are offered participation in the study.

\subsection{Description of SAFE VET intervention condition}

The SAFE VET intervention includes two protocols, Safety Planning Intervention (SPI) and Structure Follow-up (SPI-SFU) [22]. Both interventions are carried out by clinically trained study personnel working under the job title of "Acute Services Coordinators [ASC]." On occasion, the SPI was administered by other ED staff, when the ASC was unavailable. Other ED staff at the SAFE VET sites was also trained in the administration of the SPI. Study-related professional staff is employed to provide the intervention both to increase intervention fidelity and to eliminate any concern about undue burden on ED staff. The ASCs are master's level trained mental health practitioners with psychology, nursing or social work backgrounds. ASCs are fully integrated into the day-to-day operations of the ED, functioning as ancillary ED staff who also see other Veterans when not engaged in study work. 
Safety Planning Intervention. As part of a comprehensive plan to reduce suicide risk in Veterans treated in the VAMCs, Stanley and Brown [24] adapted a brief clinical intervention, the Safety Planning Intervention (SPI), for use by mental health clinicians across the VAMC behavioral health treatment spectrum. In collaboration with other VA staff, Stanley and Brown (2008) also developed a detailed manual, "Safety Plan Treatment Manual to Reduce Suicide Risk: Veteran Version," [24] and a training video through the VA Employee Education Service and a brief instructional guide, (available at http://www.mentalhealth.va.gov/docs/vasafetyplancolor.pdf).

SPI is a very brief, highly structured intervention (20-40 minutes) rooted in Cognitive Behavior Therapy in which patient and provider collaboratively develop a written, personalized safety plan for patient reference during suicidal crisis. Motivational enhancement to use the SPI during crisis and lethal means restriction are also targeted. There are six steps in the SPI, which are briefly described below. The SPI form (available at http://www.suicidesafetyplan.com/) lists these steps and thus serves as a guide for the provider and patient completing the SPI. Step one of the SPI is to list personal warning signs of an impending suicidal crisis. Step two involves determining internal coping strategies (namely, activities that may serve to distract from suicidal thoughts and urges). In step three, family members and friends who may distract from suicidal thoughts or urges as well as safe places to go during crisis are identified. Step four involves listing names and contact information of people who may help during suicidal crisis. Whereas in step three, the primary purpose of connecting to social resources is to distract, in step four, resources will be asked for help and thus patients are encouraged to include only those family members and friends with whom they feel comfortable sharing their mental 
health difficulties. Step five includes construction of a personalized list of mental health professionals and local urgent services to contact for help during suicidal crisis. During step six, ways to restrict access to identified lethal means are brainstormed and noted. Problem solving and motivational enhancement to increase the likelihood of postdischarge utilization of mental health resources (including the SPI) are also undertaken.

Structured Follow-up and Monitoring (SFU). The SFU component of SAFE VET is comprised of post-ED discharge telephone contact by the ASC who completed SPI with SAFE VET participants. Both the content and process of SFU is structured as follows: A first call is placed to the Veteran within a target tame frame of 72 hours (or up to 1 week if contact is delayed) of ED presentation. Follow-up contact includes three components: assessment including a mood check and brief suicide risk assessment; SPI review and revision, if needed; and enhancing treatment motivation through problem solving of any obstacles to treatment. If the suicidal individual is in imminent risk, immediate rescue procedures are implemented. Follow-up calls are terminated when the patient is engaged with follow-up mental health services, no longer wishes to be contacted, or has clearly refused outpatient care. ASCs attempt to contact each participant multiple times if initial efforts to reach participants are unsuccessful. Further, should a Veteran not have an outpatient appointment within 1 week of ED presentation, ASCs also complete subsequent calls (to include safety assessment, intervention, treatment motivation enhancement, and safety plan review) weekly until the Veteran attends his or her first outpatient appointment where mental health or substance use treatment is provided. SFU is discontinued once the Veteran is successfully engaged in 
outpatient treatment (defined as attending 2 sequential scheduled outpatient treatment appointments).

Specific training and supervision of the ASC on the delivery of the SAFE VET intervention is provided by Drs. Stanley and Brown through didactic workshops, videotaped demonstrations, and assigned readings. During the training phase, role-plays are also conducted to provide practice in intervention delivery and improve fidelity to the treatment. On-going weekly group supervision is also completed by a licensed Ph.D.level psychologist, well versed in SPI and SFU throughout the duration of the clinical demonstration project. Once site enrollment targets are met, the ASCs at each site will then train other ED nursing and social work staff on appropriate administration of Safety Plans and structured aftercare.

\subsection{Description of control condition: Enhanced usual care (E-CARE)}

E-CARE consists of usual care as practiced within each VAMC ED, with the addition of research-driven telephone contacts after ED discharge. Usual care generally consists of an initial assessment and history-gathering by a nurse or social worker, and a secondary evaluation and decision about need for admission by an ED physician. In most VAMC ED settings, non-physician clinical staff are responsible for any psychosocial interventions. Discharged patients are provided with either a specific outpatient appointment or information about how to seek such services if the patient declines to accept the referral at the time of discharge. Once patients leave the ED, there is usually no specific outreach by ED staff, and no routine effort is made to check if patients attended outpatient aftercare. Safety planning is not a component of routine care of suicidal patients at any of the control EDs. Therefore, the addition of baseline and follow- 
up research assessments to usual care services under SAFE VET comprises the only enhancement to usual care in this study.

\subsection{Measures}

\subsubsection{Primary outcome measures.}

The primary hypothesis for this study is that subjects who receive the SPI-SFU intervention will enact fewer suicide attempts within the first 6 months after ED discharge versus those who do not receive the intervention. Suicide attempts are classified using the Columbia Suicide-Severity Rating Scale [25]. The C-SSRS is a clinician-administered measure that assesses for suicide ideation and attempt behavior based on established definitions.

\subsubsection{Secondary outcome measures.}

We further hypothesize that subjects receiving the SPI-SFU intervention will demonstrate:

2. Reduced suicidal ideation at 6 months post-ED visit,

3. Increased suicide-related coping abilities at 6 months post-ED visit,

4. Higher overall rate of, and reduced time to, successful attendance at an outpatient mental health or chemical dependency treatment session after ED discharge.

Secondary outcome measures include:

Scale for Suicide Ideation [26]. The SSI is a 21-item interviewer-administered scale with an estimated administration time of 10 minutes. The SSI evaluates the current intensity of respondent attitudes towards death and suicide, suicidal behaviors and plans. 
Suicide-Related Coping Measure. This measure was designed for the purposes of this study and assesses participant attitudes regarding availability of and self-efficacy in using coping strategies to manage suicidal thoughts and urges.

Cornell Services Index (CSI)[27] -Modified. The CSI was shorted for use with the target population. The CSI is a clinician-administered measure to collect information on ED visits, hospitalizations, outpatient mental health and substance use treatment services received since the last assessment or the index ED visit.

Perceived Barriers to Care (PBTC) [28]-Modified. The PBTC is a self-report instrument to assess perceived barriers to mental health treatment, particularly stigmatization.

Attitudes Toward Seeking Professional Psychological Help Scale: Modified Shortened Version[29, 30]. The ATSPPH is a self-report measure to assess attitudes toward seeking help for mental health issues. Higher scores reflect more favorable attitudes toward seeking professional mental health services.

Medical records are reviewed to determine VAMC health service usage in the sixmonth period after study enrollment. Demographic information is also collected including age, gender, race, ethnicity, education, religious affiliation, relationship status, employment status, branch of military service, period of service, service connection, history of deployment and combat, and homelessness. Veterans are also asked about past diagnoses with mental disorders, current prescribed medications, and family history of psychiatric illness or suicidal behavior.

\subsection{Training, supervision, and adherence of study assessors}

At one, three, and six months post-enrollment, study personnel telephone study 
participants and administer rating scales . All interviewers are at least Master's level clinicians who have received extensive training in the administration of structured interviews. To prevent rater drift, supervision, as well as refresher training, as indicated, is ongoing, and audio-taped administrations of assessments are periodically reviewed.

\subsection{Safety considerations}

The VA Institutional Review Boards (IRBs) at all study sites provide regulatory oversight for this study. To minimize IRB concerns about patient safety for high risk participants, we tried to anticipate the safety issues that occur when dealing with individuals at imminent risk for suicidal behavior during the assessment interviews. Thus, we proposed comprehensive procedures if rescue was needed, including immediate access to senior clinical investigators, warm hand-off procedures and close connections with the national Veterans Crisis Line (see section 2.11).

\subsubsection{Potential risks}

Risks from participation in this study are generally attendant to those associated with participation in routine psychiatric interviews. Additional potential risks include violation of confidentiality and possible negative reactions to the assessment, including distress due to discussion of sensitive subject matter. Participants who receive SPI-SFU may spend time participating in an intervention that is not helpful for their emotional and behavioral difficulties.

\subsubsection{Potential benefits}

While there are no proven benefits to participation, patients hospitalized for selfinjurious behavior who receive supportive contact from mental health professionals in the weeks to months following hospital discharge may be less likely to self-injure as 
frequently as patients who do not. Subjects may also benefit from the thorough evaluations and resources (including information about local emergency services) they receive throughout their study participation. The systematic, comprehensive monitoring of suicidal thoughts and behavior completed at every assessment throughout the course of the study increases the likelihood that elevated suicide risk will be detected early and that appropriate interventions will be received.

\subsection{High risk management procedures}

The procedures outlined in this section protect against or minimize the potential risks of study participation. During the informed consent procedure, the limits of confidentiality are explained to all participants. Specifically, confidentiality may be broken in cases of: (1) imminent risk for self-harm; (2) imminent risk for harming others; and (3) abuse (neglect, physical, and sexual) of an identified minor or identified person over the age of 65 .

If, based on clinical impression, a participant appears to be at elevated but not imminent risk of suicide during a follow-up phone assessment, two steps occur. First, research staff initiate a transfer to the Veterans Crisis Line (1-800-273-8255, press 1) in Canandaigua, NY. Second, research raters contact the PI or a senior mental health professional - Ph.D. or M.D. level - to whom responsibility has been delegated, to notify them of the situation. Assessors have emergency contact information available at all times for the PI or designee and emergency services (e.g. Veterans Crisis Line). As clinically indicated, assessors stay on the telephone with the participant until they are able to consult with a site principal investigator or designee, determine next steps, and followthrough with them. Assessors receive training and supervision on suicide risk assessment 
and suicide crisis procedures. These procedures include reporting these incidents to the site principal investigator and initiating appropriate emergency response interventions as indicated. In addition, the IRBs at participating hospitals and affiliated universities are informed of any incidents requiring emergency interventions.

\subsubsection{Adverse Events}

All assessments are documented in research files. All members of the research team are familiar with procedures for identifying and reporting possible Adverse Events. As this is a multisite study, IRB regulations regarding Adverse Events vary across sites. Severe and minor adverse events are reported to IRB sites per current policies outlined in this attached document. At all sites, adverse events are reviewed by the site principal investigator or designee to establish the level of seriousness. During this process the site principal investigator or designee will classify the attribution of the adverse event (e.g., definitely, probably, possibly related; unlikely or unrelated) and grade the severity of the event, utilizing the FDA's definition of serious adverse events, on a 6-point scale $(0=$ no adverse event or within normal limit; $1=$ mild; $2=$ moderate; $3=$ severe; $4=$ life-threatening; $5=$ fatal). Serious unanticipated or anticipated adverse events are reported in a timely manner to the IRBs.

\subsubsection{Discontinuation from the study}

Participants are informed that they have the right to discontinue at any point in the study.

2.12. Planned statistical analysis

\subsubsection{General}


A comparison of baseline sample characteristics between E-CARE and SPI-SFU subjects will be completed using t-tests or the non-parametric equivalent (i.e., the Wilcoxon rank sum test) or Chi-square tests, depending on the type (continuous or dichotomous) and distribution (normal or non-normal) of the data. These analyses will not only describe the sample, but also identify covariates for inclusion in the analyses testing SPI-SFU effectiveness.

\subsubsection{Between group differences in primary outcomes}

Repeated-measures mixed effect regression analysis will be used to test for differences in outcomes between SPI-SFU and E-CARE recipients. Specifically, the generalized linear mixed-effects model (GLMM) approach will be used except for nonnormal data where a generalized estimating equation (GEE) approach will be used. Treatment group (binary indicator), time, and time by treatment group interaction will be included as predictors. Covariates will be added to control for any baseline differences between groups. Because of the naturalistic context of the intervention, between group differences in VAMC health care service usage during the 6-month follow-up period will also be assessed and considered in subsequent analyses when such differences are found. For the GLMM and GEE analyses, model selection procedures will be applied to find the most parsimonious model using Akaike's information criterion (AIC) as a guide for model selection [31].

The risk of suicide attempt and time to outpatient mental health treatment in the 6 months after the initial assessment will be evaluated using Cox proportional hazards regression analysis with treatment group as the independent variable. Possible confounding effects, i.e., those baseline variables on which groups differed, will be 
controlled. The false discovery rate (FDR) will be used to control for type I error when testing multiple hypotheses. FDR measures the number of false significant results and thus, addresses the main problem with other multiple comparison tests, such as the Bonferroni's method, which are often too conservative [32].

\subsubsection{Missing data}

We will examine the nature of missing data and use appropriate statistical methods to analyze the data. If data are not missing completely at random, the weighted GEE (WGEE) approach will be applied to overcome the limitation of GLMM and GEE.

\subsubsection{Power analysis to determine sample size}

Power analyses were conducted using rates of repeat suicide attempts reported for ED patients enrolled in trials comparing the efficacy of a psychosocial intervention to enhanced usual care $[33,34]$. In these studies, rates of repeat suicide attempts in the enhanced usual care arm have varied from $17 \%$ over $6-12$ months to $35 \%$ to $38 \%$ over 24-months. Thus, assuming a 20\% dropout rate and the planned enrollment of 300 subjects per condition, the current study would have $80 \%$ power to detect a $10 \%$ difference in the proportion of patients who make a suicide attempt during the 6 month follow-up period.

\section{Discussion}

The importance of suicide prevention efforts for Veterans has received enormous attention in the popular press, Congress and the VA. Evidence suggests that suicide rates are historically higher among Veterans who are enrolled in the VA versus the general population $^{2}$. Additionally, Veterans who survive a suicide attempt remain at substantially elevated risk of dying by suicide [7]. As such, an important goal of this trial is to assess 
the effectiveness of SPI-SFU for reducing suicide attempt risk among suicidal, EDdischarged Veterans who seek care in VAMC ED settings. This study has several components that may impact generalizability and sustainability. The role of ASC was created for this project to minimize undue burden on ED staff and to assure fidelity to the intervention. Further, a randomized, rater-blinded controlled trial would have been a preferred approach. However, in this project such an approach is not feasible because the intervention being studied has already been introduced into the treatment environment. Matching very similar EDs involved in a staggered rollout, where the intervention had yet to be introduced, provides an alternative opportunity to examine clinical effectiveness.

\section{Summary}

Few suicide prevention interventions have been designed to work seamlessly within and across VA ED settings. This represents a significant gap in service provision. This study aims to develop and SPI-SFU in and across VA facilities, setting the stage for rapid dissemination of the intervention across this system if the effectiveness is established. Additionally, since substantial portions of Veterans receive emergency services in the civilian healthcare sector, it is important to note that the intervention under development will be readily adaptable for administration to Veterans treated in general hospital EDs as well. 


\section{Funding source}

Financial support for this research has been provided to the Henry Jackson Foundation for the Advancement of Military Medicine, by the Military Operational Medicine Research Program of the Department of Defense (W81XWH-09-2-0129).

\section{Disclaimer}

The views expressed in this article are those of the authors and do not reflect the official policy of the Department of Defense (DoD), the Department of Veterans Affairs (VA), and/or the United States Government. 
Figure 1:

\section{SAVE VET Study Design}
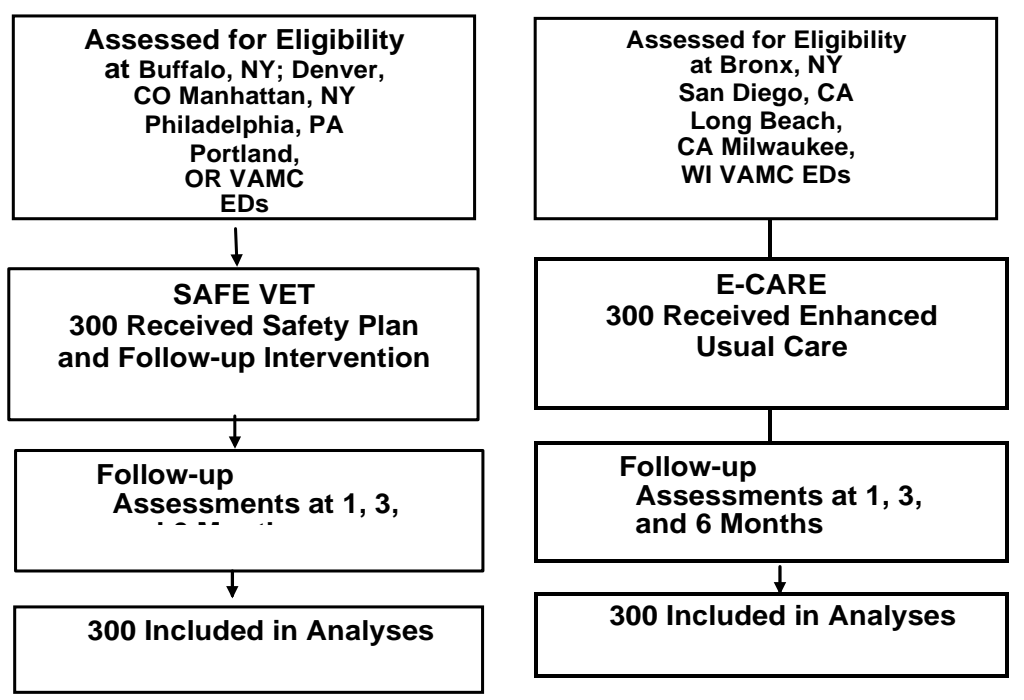


\section{$\underline{\text { References }}$}

[1] CDC. Web-Based Injury Statistics Query and Reporting System (WISQARS), Fatal Injury Reports, 2011. 2014.

[2] McCarthy JF, Valenstein M, Kim HM, Ilgen M, Zivin K, Blow FC. Suicide Mortality Among Patients Receiving Care in the Veterans Health Administration Health System. Am J Epidemiol. 2009;169:1033-8.

[3] Kaplan MS, Huguet N, McFarland BH, Newsom JT. Suicide among male veterans: a prospective population-based study. J Epidemiol Community Health. 2007;61:619-24. [4] Katz IR, McCarthy JF, Ignacio RV, Kemp J. Suicide Among Veterans in 16 States, 2005 to 2008: Comparisons Between Utilizers and Nonutilizers of Veterans Health Administration (VHA) Services Based on Data From the National Death Index, the National Violent Death Reporting System, and VHA Administrative Records. Am J Public Health. 2012;102:S105-S10.

[5] Kemp J, Bossarte R. Suicide Data Report, 2012. Department of Veterans Affairs, Mental Health Services, Suicide Prevention Program; 2012.

[6] Crosby AE, Han B, Ortega LAG, Parks SE, Gfroerer J. Suicidal Thoughts and Behaviors Among Adults Aged $\geq 18$ Years --- United States, 2008-2009. Morbidity and Mortality Weekly Report (MMWR). 2011;60:1-22.

[7] Wiebe D, Weiner J, Conigliaro J, Richmond T, Branas C. Follow-up study of US military Veterans following a suicide attempt: Mortality and causes of death. 8th World Conference on Injury Prevention \& Safety Promotion. Durban, South Africa 2006. [8] Larkin GL, Smith RP, Beautrais AL. Trends in US emergency department visits for suicide attempts, 1992-2001. Crisis. 2008;29:73-80.

[9] Natioanl Center for Health Statistics. Health, United States, 2013: With Special Feature on Prescription Drugs. Hyattsville, MD 2014.

[10] McCraig L, Nawar E. National Hospital Ambulatory Medical Care Survey: 2004 Emergency Department Summary (No. 372). In: U.S. Department of Health and Human Services CDC National Center for Health Statistics, editor. Hyattsville, MD2006. [11] Kurz A, Moller H. Help-seeking behavior and compliance of suicidal patients. Psychiatr Prax. 1984;11:6-13.

[12] Olfson M, Marcus SC, Bridge JA. Focusing suicide prevention on periods of high risk. JAMA. 2014;311:1107-8.

[13] APA Practice Guideline for the Assessment and Treatment of Patients with Suicidal Behaviors 2003.

[14] Van Heeringen C, Jannes S, Buylaert W, Henderick H, De Bacquer D, Van Remoortel J. The management of non-compliance with referral to out-patient after-care among attempted suicide patients: a controlled intervention study. Psychol Med. 1995;25:963-70.

[15] Suominen K, Lönnqvist J. Determinants of psychiatric hospitalization after attempted suicide. Gen Hosp Psychiatry. 2006;28:424-30.

[16] Jauregui J, Martinez M, Rubio G, Santo-Domingo J. Patients who attempted suicide and failed to attend mental health centres. Eur Psychiatry. 1999;14:205-9.

[17] Currier GW, Fisher SG, Caine ED. Mobile crisis team intervention to enhance linkage of discharged suicidal emergency department patients to outpatient psychiatric services: a randomized controlled trial. Acad Emerg Med. 2010;17:36-43. 
[18] Krulee DA, Hales RE. Compliance with psychiatric referrals from a general hospital psychiatry outpatient clinic. Gen Hosp Psychiatry. 1988;10:339-45.

[19] Stanley B, Brown GK. Safety Planning Intervention: A brief intervention to mitigate suicide risk. Cognitive and Behavioral Practice. 2012;19:256-64.

[20] Cooper J, Kapur N, Webb R, Lawlor M. Suicide After Deliberate Self-Harm: A 4-

Year Cohort Study. The American Journal of Psychiatry. 2005;162:297-303.

[21] National Center for Health Statistics, Prevention. Data \& Statistics Fatal Injury

Report for 2013.

[22] Knox KL, Stanley B, Currier GW, Brenner L, Ghahramanlou-Holloway M, Brown

G. An Emergency Department-Based Brief Intervention for Veterans at Risk for Suicide

(SAFE VET). Am J Public Health. 2012;102:S33-S7.

[23] Ghahramanlou-Holloway M, Brown GK, Currier GW, Brenner L, Knox KL,

Grammer G, et al. Safety Planning for Military (SAFE MIL): Rationale, design, and safety considerations of a randomized controlled trial to reduce suicide risk among

psychiatric inpatients. Contemp Clin Trials. 2014;39:113-23.

[24] Stanley B, Brown GK. Safety Plan Treatment Manual to Reduce Suicide Risk:

Veteran Version. Washington, D.C.: United States Department of Veterans Affairs; 2008. [25] Posner K, Brent D, Lucas C, er al. The Columbia-Suicide Severity Rating Scale:

New York State Psychiatric Institute; 2008.

[26] Beck AT, Kovacs, M., \& Weissman, A. Assessment of suicidal intention: The Scale for Suicide Ideation. J Consult Clin Psychol. 1979;47:343-52.

[27] Sirey JA, Meyers BS, Teresi JA, Bruce ML, Ramirez M, Raue PJ, et al. The Cornell

Service Index as a measure of health service use. Psychiatr Serv. 2005;56:1564-9.

[28] Hoge C, Castro C, Messer S, McGurk D, Cotting D, Koffman R. Combat duty in

Iraq and Afghanistan, mental health problems, and barriers to care. N Engl J Med. 2004;351:13 - 22 .

[29] Fischer EH, Turner JI. Orientations to seeking professional help: development and research utility of an attitude scale. J Consult Clin Psychol. 1970;35:79.

[30] Komiti A, Judd F, Jackson H. The influence of stigma and attitudes on seeking help from a GP for mental health problems. Soc Psychiatry Psychiatr Epidemiol. 2006;41:73845 .

[31] Akaike H. Likelihood of a model and information criteria. Journal of Econometrics. 1981;16:3-14.

[32] Aickin M, Gensler H. Adjusting for multiple testing when reporting research results: the Bonferroni vs Holm methods. Am J Public Health. 1996;86:726-8.

[33] Brown GK, Ten Have T, Henriques GR, Xie SX, Hollander JE, Beck AT. Cognitive therapy for the prevention of suicide attempts: A randomized controlled trial. JAMA. 2005;294:563-70.

[34] Fleischmann A, Bertolote JM, Wasserman D, De Leo D, Bolhari J, Botega NJ, et al. Effectiveness of brief intervention and contact for suicide attempters: a randomized controlled trial in five countries. Bull World Health Organ. 2008;86:703-9. 